\title{
QUALIDADE SENSORIAL DE NÉCTAR DE CAMU- CAMU PRODUZIDO COM POLPA PASTEURIZADA PÓS-EMBALAGEM ARMAZENADA EM DIFERENTES TEMPERATURAS
}

\author{
SAMUEL ZANATTA* \\ PAULA PORRELLI M. DA SILVA** \\ MARIA HELENA COSTA*** \\ MARTA HELENA FILLET SPOTO****

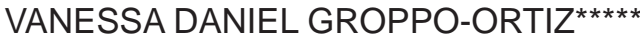

\begin{abstract}
O objetivo deste trabalho foi verificar a qualidade sensorial do néctar de camu-camu elaborado com polpa pasteurizada pósembalagem, utilizando-se bolsas plásticas de alta barreira, frente ao armazenamento refrigerado $\left(5^{\circ} \mathrm{C}\right)$, congelado $\left(-18{ }^{\circ} \mathrm{C}\right)$ e à temperatura ambiente $\left(28^{\circ} \mathrm{C}\right)$ durante 45 dias. As amostras foram pasteurizadas em tanque aquecido a $75{ }^{\circ} \mathrm{C}$ durante 1 minuto e resfriadas imediatamente. Os resultados de aceitação global indicaram que os néctares foram bem aceitos, sendo a polpa congelada classificada como "gostei muito" e as demais como "gostei moderadamente" (temperatura ambiente e refrigerada). A comercialização da polpa de camu-camu torna-se interessante devido ao alto teor de ácido ascórbico que a fruta apresenta e limitação de consumo na forma in natura em razão de sua elevada acidez. A polpa produzida pode ser utilizada na elaboração de bebidas alimentícias, preparo de sorvetes, geleias, doces e licores.
\end{abstract}

PALAVRAS-CHAVE: Myciaria dubia; CAMU-CAMU; NÉCTAR; VITAMINA C; POLIPROPILENO DIFERENCIADO.

* Mestrando em Química na Agricultura e no Ambiente, Centro de Energia Nuclear na Agricultura, Universidade de São Paulo (CENA/USP), Piracicaba, SP (e-mail: zanatta@cena.usp.br).

** Doutora em Ciências, Centro de Energia Nuclear na Agricultura e Ambiente, CENA/USP, Piracicaba, SP (e-mail: pporrelli@uol.com.br).

*** Engenheira de Alimentos, Departamento de Agroindústria, Alimentos e Nutrição, Escola Superior de Agricultura "Luiz de Queiroz", Universidade de São Paulo (ESALQ/USP), Piracicaba, SP (e-mail mahe_costa@hotmail.com).

**** Professora, Doutora em Tecnologia Nuclear, Departamento de Agroindústria, Alimentos e Nutrição, ESALQ/USP, Piracicaba, SP (e-mail: martaspoto@usp.br).

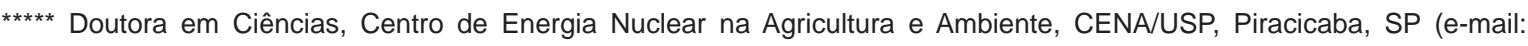
vanessa_groppo@yahoo.com.br). 


\section{INTRODUÇÃO}

O camu-camu (Myrciaria dubia), fruto silvestre nativo da Amazônia, pertence à família Myrtaceae. Ocorre nas margens de rios e lagos da Amazônia, sendo propagado por sementes. As sementes extraídas de frutos no mesmo estágio de maturação proporcionam maior uniformidade na germinação. A planta, típica de clima tropical quente e úmido, apresenta boas características agronômicas. Desenvolve-se bem em locais com temperatura média entre $22^{\circ} \mathrm{C}$ a $28^{\circ} \mathrm{C}$, suportando temperaturas mínimas e máximas em torno de $17^{\circ} \mathrm{C}$ a $35^{\circ} \mathrm{C}$ e umidade relativa (UR) de 70 até 95 \%. O potencial econômico do camu-camu pode colocá-lo no mesmo nível alcançado por outras frutas tradicionais da região amazônica, como o açaí e o cupuaçu (INPA, 2011, ARRUDA et al., 2011).

Os frutos dificilmente são consumidos in natura devido à elevada acidez e ao amargor da casca, mas assumem importância na alimentação pelo alto valor nutricional. Além de rico em ácido ascórbico, o camu-camu contém elevada concentração de antocianinas evidenciada pela cor vermelho-púrpura do fruto (MAEDA et al., 2007; INPA, 2011).

As características que limitam o consumo de camu-camu (elevada acidez e baixo pH) são desejáveis no processamento de polpa e suco (FRANCO e SHIBAMOTO, 2000). Assim, uma das maneiras para aumentar o consumo do camu-camu consiste em agregar valor ao produto, mediante processamento e preparo de polpa, suco ou geleia.

O processamento do fruto torna-se necessário em razão de seu baixo tempo de vida útil in natura, que inviabiliza sua comercialização. Além disso, danos ocorridos aos tecidos e células durante o manuseio e/ou transporte do fruto provocam perda de peso por ressecamento, causando desinteresse do consumidor (ARÉVALO, KIECKBUSCH e QUAST, 2006).

O tipo de embalagem empregada em produtos alimentícios armazenados influencia suas reações metabólicas, diminuindo seu ritmo por meio da modificação do microclima criado em seu interior, constituindo barreira que impede ou dificulta o contato entre o ambiente externo e 0 produto (GARCIA, PADULA e SARANTÓPOULOS, 1989). As polpas de frutas são comercializadas geralmente em embalagens flexíveis (bolsas plásticas de polietileno) ou em embalagens cartonadas assépticas, devido à facilidade de manuseio e à proteção contra oxidações, evitando possíveis alterações das características sensoriais do produto (BRUNINI, DURIGAN e OLIVEIRA, 2002).

O objetivo deste trabalho foi verificar a qualidade sensorial do néctar de camu-camu produzido com polpa pasteurizada pós-embalagem, acondicionada em bolsa plástica de alta barreira, frente ao armazenamento refrigerado, congelado e à temperatura ambiente.

\section{MATERIAL E MÉTODOS}

\subsection{PROCESSAMENTO}

Os frutos utilizados no experimento são provenientes da 'Fazenda Eldorado' na região

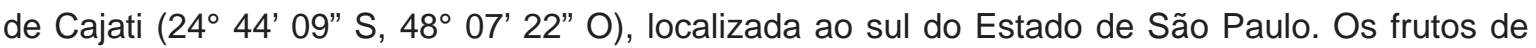
camu-camu, selecionados quanto à dimensão, volume, cor, aparência e sanidade, foram imersos em solução de hipoclorito de sódio (200 ppm) por 15 minutos, lavados com água e despolpados em despolpador de aço inox. Acondicionou-se a polpa manualmente em embalagem de alta barreira $(100 \mathrm{~mL})$. A característica principal dessa embalagem (que oferece resistência à temperatura de até $121{ }^{\circ} \mathrm{C}$ por 45 minutos, pH 3,5 e vácuo) reside na sua composição em multicamadas sobrepostas: PET $15 \mathrm{U}$ - politereftalato de etila (poliéster); OPA 15u - poliamida biorientada (nylon) e CPP 70u polipropileno obtido por extrusão (PP Cast).

As embalagens foram seladas e levadas ao tanque para pasteurização a $75{ }^{\circ} \mathrm{C}$ por 1 minuto, de acordo com Silva (2012). Para assegurar a uniformidade da temperatura no tanque de pasteurização e temperatura adequada no interior das embalagens utilizou-se Termopar, da marca 
Novus, com quatro sensores. As amostras foram resfriadas imediatamente após a pasteurização em tanque com água a $40^{\circ} \mathrm{C}$ e, posteriormente armazenadas sob diferentes condições: refrigeração a $5^{\circ} \mathrm{C}$ (R5); congelamento a $-18^{\circ} \mathrm{C}(\mathrm{C} 18)$ e em estufa incubadora a $28^{\circ} \mathrm{C}(\mathrm{A} 28)$.

\subsection{ANÁLISES MICROBIOLÓGICAS}

Efetuaram-se as avaliações microbiológicas exigidas pela RDC n¹2 da ANVISA (BRASIL, 2001) para polpa de frutas concentradas ou não, com ou sem tratamento térmico, refrigeradas ou congeladas.

\subsubsection{Número Mais Provável (NMP) de coliformes totais e termotolerantes}

As análises microbiológicas foram realizadas tendo como parâmetro a RDC $n^{0} 12$, de 02 de janeiro de 2001, que estabelece o regulamento técnico sobre padrões microbiológicos para alimentos (BRASIL, 2001). Efetuou-se a determinação do NMP de coliformes totais e termotolerantes utilizando o kit Simplate (BIOCONTROL $\left.{ }^{\circledR}, 2013\right)$.

\subsubsection{Pesquisa da presença de Salmonella spp}

Para a detecção de Salmonella sp. utilizou-se o kit "1-2 test", fabricado pela Biocontrol ${ }^{\circledR}$, de acordo com a AOAC (2000).

\subsection{ANÁLISE SENSORIAL}

As polpas pasteurizadas e armazenadas em diferentes temperaturas foram avaliadas sensorialmente após $1,15,30$ e 45 dias do processamento, sendo selecionados aleatoriamente para cada sessão sensorial trinta julgadores não treinados, entre estudantes e funcionários do Departamento de Agroindústria, Alimentos e Nutrição da Escola Superior de Agricultura "Luiz de Queiroz" (ESALQ/USP).

Preparou-se o néctar de camu-camu na proporção de $17 \%$ de polpa, 17,5 \% de açúcar e $65,5 \%$ de água (MAEDA et al., 2006), sendo acondicionados $30 \mathrm{~mL}$ do néctar em copos plásticos brancos com $50 \mathrm{~mL}$ de capacidade, codificados com números de três dígitos, segundo delineamento experimental em Quadrados Latinos (3x3) (COCHRAN e COX, 1992).

Orientou-se cada julgador a provar as amostras da esquerda para a direita, tomando água entre as amostras e a indicar na ficha com escala hedônica estruturada de nove pontos (Figura 1) o quanto gostou ou desgostou (9 = gostei muitíssimo; 1 = desgostei) de cada amostra para os atributos aparência, sabor, textura, aroma e impressão global (MEILGAARD, CIVILLE e CARR, 1999).

O projeto de pesquisa foi aprovado pelo Comitê de Ética em Pesquisa com Seres Humanos da ESALQ/USP, sob o Protocolo n 64.

\subsection{ANÁLISE ESTATÍSTICA DOS RESULTADOS}

Adotou-se delineamento experimental inteiramente ao acaso, relacionando três tratamentos de néctar de camu-camu $\left(-18{ }^{\circ} \mathrm{C}, 5^{\circ} \mathrm{C}\right.$ e $\left.28^{\circ} \mathrm{C}\right)$ e quatro períodos de armazenamento $(1,15,30 \mathrm{e}$ 45 dias) com trinta repetições, que constituíam os julgadores não treinados.

Os resultados obtidos nos testes sensoriais foram submetidos à análise de variância (ANOVA) pelo Teste F e comparação das médias pelo Teste de Tukey (5\%), utilizando-se o programa Statistical Analysis System, versão 9.2 (SAS, 2005).

Para avaliar a interdependência entre as variáveis sensoriais calculou-se a matriz de 
correlação a partir dos dados autoescalonados. Foram selecionados os coeficientes de correlação com índices maiores que 0,70, considerados acentuados de acordo com Sounis (1975). Os coeficientes apresentados são estatisticamente significantes acima do nível de $95 \%$ de confiança. Realizou-se a análise de correlação dos dados sensoriais usando o programa estatístico PC-ORD (MCCUNE e MEFFORD, 1999).

Nome: Data:

Você está recebendo três amostras de suco de camu-camu. Avalie cada uma das amostras codificadas e use a escala abaixo para indicar o quanto você gostou ou desgostou de cada uma.

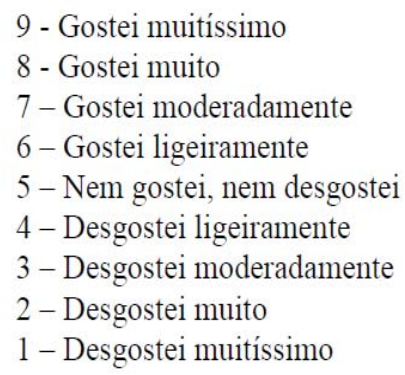

\begin{tabular}{|l|l|l|l|l|}
\hline Amostra & Cor & Aroma & Sabor & Impressão Global \\
\hline & & & & \\
\hline & & & & \\
\hline & & & & \\
\hline
\end{tabular}

Mais gostou e menos gostou. Por quê?

\section{FIGURA 1 - MODELO DA FICHA DE ANÁLISE SENSORIAL UTILIZANDO ESCALA HEDÔNICA DE NOVE PONTOS}

O conjunto de dados também foi submetido aos tratamentos matemáticos e estatísticos multivariados, como análises de componentes principais tipo - $\mathrm{R}$ e representação gráfica (SOUNIS, 1975; HARPER e KOWALSKI, 1979; SCARMINIO, 1989).

\section{RESULTADOS E DISCUSSÃO}

\subsection{ANÁLISE MICROBIOLÓGICA}

Quanto à contagem microbiológica para coliformes a $45^{\circ} \mathrm{C}$ e pesquisa de Salmonella sp., a polpa in natura apresentou-se de acordo com os parâmetros recomendados pela RDC $n^{\circ} 12$ da ANVISA (BRASIL, 2001).

Neves et al. (2007) avaliaram o processo de pasteurização (95 ${ }^{\circ} \mathrm{C}$ por 1 minuto) de polpa de manga durante 28 dias. Obtiveram resultados satisfatórios para esterilização microbiológica, perfil físico e químico ( $\mathrm{pH}$, acidez titulável e sólidos solúveis) e preferência sensorial.

Em estudos com polpa de açaí, Sousa et al. (2006) avaliaram o produto in natura; pasteurizado a 70, 80, 90 e $100{ }^{\circ} \mathrm{C}$ durante 1, 5, 10 minutos; e submetido à fervura por 1,2 e 5 minutos. Verificaram maior eficiência do suco pasteurizado a $90{ }^{\circ} \mathrm{C}$ por 10 minutos e o fervido por 1 
minuto na destruição de micro-organismos após conservação a $5{ }^{\circ} \mathrm{C}$.

\subsection{AVALIAÇÃO SENSORIAL DOS NÉCTARES DE CAMU-CAMU}

O aroma dos néctares R5, C18, A28, durante os 30 dias de avaliação não apresentou diferença significativa, sendo bem aceito pelos julgadores (notas entre "gostei muito" e "gostei moderadamente"). Aos 45 dias de armazenamento, a polpa A28 recebeu menor nota ("gostei ligeiramente") dos julgadores (Tabela 1).

A polpa A28 recebeu as menores notas para o atributo cor, devido degradação dos pigmentos vermelhos (as antocianinas), que tornou o produto menos atraente quando comparado às polpas dos demais tratamentos.

As polpas R5 e C18 mantiveram a coloração até o último período de avaliação, obtendo ótima aceitação entre os julgadores que as classificaram entre os termos "gostei moderadamente" e "gostei muito" (Tabela 1). Resultado semelhante foi encontrado por Silva et al. (2008) estudando néctar de caju. A média do atributo coloração permaneceu entre "gostei moderadamente" e "gostei muito", enquadrando-se na faixa de variação de aceitação.

Para o atributo sabor, no período 1, as polpas se mostravam em igual patamar de aceitação ("gostei muito"). A partir do período 15, as polpas A28 e R5 apresentaram variações nos valores médios atribuídos pelos julgadores. A aceitação da polpa A28 diminuiu de acordo com o tempo ("nem gostei, nem desgostei"), enquanto a aceitação da polpa C18 se manteve mais estável. Todas as polpas mostraram decréscimo de aceitação no período 45 (Tabela 1).

A impressão global reflete a junção de todos os atributos para cada amostra. A polpa C18, classificada pelo termo hedônico "gostei muito", não sofreu interferência do período de armazenamento, sendo considerada bem aceita pelos julgadores. As polpas A28 e R5 obtiveram menor aceitação a partir do período 15, ficando suas médias entre os termos "não gostei, nem desgostei" e "gostei moderadamente" (Tabela 1).

Segundo Oliveira et al. (2011), a pasteurização ( $88^{\circ} \mathrm{C} / 10$ segundos) de polpa de umbu (S. tuberosa) não exerceu efeito sobre o sabor e o aroma porque não houve diferença significativa $(p<0,05)$ entre a polpa processada e a controle. No entanto, foi percebido sabor amargo na polpa de umbu pasteurizada a $96{ }^{\circ} \mathrm{C}$ por 30 segundos.

Deve-se a aceitação da polpa C18 pelos julgadores ao congelamento, que manteve a coloração e o sabor característico do fruto e também à concentração da formulação. Maeda et al. (2006) verificaram tendência de ótima aceitabilidade do néctar de camu-camu pelos julgadores quando utilizaram concentração de polpa que tornou seu sabor amargo e adstringente imperceptível, mas adotando concentração suficiente para manter o sabor característico do fruto.

Silva e Silva (2000), ao pasteurizarem purê de cupuaçu a $70^{\circ} \mathrm{C}$ e $90^{\circ} \mathrm{C}$ por 5 minutos, verificaram que o produto reteve grande parte das propriedades sensoriais originais da fruta. Nenhuma diferença estatística foi notada para o sabor, o aroma e a cor das amostras entre as duas temperaturas estudadas durante o período de armazenamento avaliado.

Bastos e colaboradores (2008) estudaram polpa de taperebá (Spondias mombin) pasteurizada ( $85^{\circ} \mathrm{C} / 3$ minutos) e verificaram elevada aceitação do produto ( $80 \%$ em média), independentemente do período de armazenamento. Ainda, os julgadores não conseguiram identificar a amostra pasteurizada quando comparada ao controle.

O resultado encontrado na análise sensorial de néctar de camu-camu mostrou a aceitação dos julgadores por produto com sabor exótico. Resultados semelhantes foram obtidos por Mattieto, Lopes e Menezes (2007) ao estudarem néctar misto de umbu e cajá submetido à pasteurização térmica $\left(90^{\circ} \mathrm{C} / 60 \mathrm{~s}\right)$, que indicaram boa aceitação em relação à impressão global $(84,76 \%)$ e intenção de compra $(90,62 \%)$ do produto. Tal fato justifica os estudos de processamento e armazenamento de outros frutos com características exóticas de sabor e aroma e a elaboração de novos produtos, cujo mercado vem ganhando maior dimensão. 
TABELA 1 - AROMA, COR, SABOR E IMPRESSÃO GLOBAL DO NÉCTAR DE CAMU-CAMU

ELABORADO A PARTIR DE POLPA PASTEURIZADA PÓS-EMBALAGEM, ARMAZENADA

EM DIFERENTES TEMPERATURAS (VALORES MÉDIOS, $\pm \mathrm{DP}, \mathrm{N}=\mathbf{3 0}$ )

\begin{tabular}{ccccc}
\hline \multicolumn{5}{c}{ AROMA } \\
\hline \multicolumn{5}{c}{ Período (dias) } \\
\hline Tratamentos & 1 & 15 & 30 & 45 \\
\hline A28 & $6,40 \pm 1,8 \mathrm{Aa}$ & $5,83 \pm 1,8 \mathrm{Aa}$ & $5,83 \pm 1,6 \mathrm{Aa}$ & $4,80 \pm 2,1 \mathrm{Ab}$ \\
C18 & $6,42 \pm 1,7 \mathrm{Aa}$ & $6,73 \pm 1,3 \mathrm{Aa}$ & $7,17 \pm 1,5 \mathrm{Aa}$ & $7,17 \pm 1,3 \mathrm{Aa}$ \\
R5 & $6,77 \pm 1,8 \mathrm{Aa}$ & $6,13 \pm 1,5 \mathrm{Aa}$ & $6,60 \pm 1,8 \mathrm{Aa}$ & $6,96 \pm 1,7 \mathrm{Aa}$ \\
\hline CV = 25,99 \% & \multicolumn{5}{c}{ COR } \\
\hline \multicolumn{5}{c}{ Período (dias) } \\
\hline Tratamentos & 1 & 15 & 30 \\
\hline A28 & $5,87 \pm 1,7 \mathrm{Aa}$ & $6,37 \pm 2,2 \mathrm{Aab}$ & $6,13 \pm 2,1 \mathrm{Ab}$ & $5,87 \pm 1,9 \mathrm{Ab}$ \\
C18 & $6,39 \pm 2,2 \mathrm{Aab}$ & $7,53 \pm 1,2 \mathrm{Aa}$ & $7,73 \pm 1,6 \mathrm{Aa}$ & $7,80 \pm 1,3 \mathrm{Aa}$ \\
R5 & $7,50 \pm 1,3 \mathrm{Ab}$ & $5,83 \pm 1,6 \mathrm{Bb}$ & $6,67 \pm 1,9 \mathrm{ABab}$ & $5,83 \pm 2,0 \mathrm{Bb}$ \\
\hline
\end{tabular}

$\mathrm{CV}=26,67 \%$

\section{SABOR}

\begin{tabular}{ccccc}
\hline \multicolumn{5}{c}{ Período (dias) } \\
\hline Tratamentos & 1 & 15 & 30 & 45 \\
\hline A28 & $6,70 \pm 1,9 \mathrm{Aa}$ & $5,20 \pm 2,3 \mathrm{ABb}$ & $5,17 \pm 2,2 \mathrm{ABb}$ & $4,33 \pm 2,2 \mathrm{Bb}$ \\
C18 & $7,06 \pm 1,5 \mathrm{Aa}$ & $7,47 \pm 1,5 \mathrm{Aa}$ & $7,70 \pm 1,3 \mathrm{Aa}$ & $7,17 \pm 1,7 \mathrm{Aa}$ \\
R5 & $7,43 \pm 1,6 \mathrm{Aa}$ & $6,23 \pm 1,9 \mathrm{ABab}$ & $7,30 \pm 1,6 \mathrm{ABa}$ & $5,76 \pm 2,4 \mathrm{Bab}$ \\
\hline $\mathrm{CV}=28,94 \%$ & & & & \\
\hline
\end{tabular}

IMPRESSÃO GLOBAL

\begin{tabular}{ccccc}
\hline \multicolumn{5}{c}{ Período (dias) } \\
\hline Tratamentos & 1 & 15 & 30 & 45 \\
\hline A28 & $6,63 \pm 1,5 \mathrm{Aa}$ & $5,47 \pm 2,1 \mathrm{ABb}$ & $5,70 \pm 1,8 \mathrm{ABb}$ & $4,53 \pm 1,9 \mathrm{Bb}$ \\
C18 & $6,84 \pm 1,5 \mathrm{Aa}$ & $7,30 \pm 1,3 \mathrm{Aa}$ & $7,73 \pm 1,2 \mathrm{Aa}$ & $7,17 \pm 1,4 \mathrm{Aa}$ \\
R5 & $7,40 \pm 1,4 \mathrm{Aa}$ & $6,10 \pm 1,6 \mathrm{ABab}$ & $7,03 \pm 1,5 \mathrm{ABab}$ & $5,90 \pm 1,8 \mathrm{Bab}$ \\
\hline
\end{tabular}

$\mathrm{CV}=24,82 \%$

Nota: Médias seguidas pela mesma letra maiúscula na linha e minúscula na coluna não diferem entre si pelo teste de Tukey ao nível de $5 \%$ de probabilidade. $\mathrm{DP}=$ desvio padrão das médias; $\mathrm{n}=$ número de repetições utilizadas; $\mathrm{CV}=$ coeficiente de variação; $\mathrm{A} 28$ = polpa de camu-camu armazenada a $28^{\circ} \mathrm{C} ; \mathrm{C} 18$ = polpa de camu-camu armazenada sob congelamento $\left(-18{ }^{\circ} \mathrm{C}\right) ; \mathrm{R} 5$ = polpa de camu-camu armazenada sob refrigeração $\left(5^{\circ} \mathrm{C}\right)$.

\subsection{SIMILARIDADES E DIFERENÇAS ENTRE OS NÉCTARES}

Os dados gerados permitiram o desenvolvimento de gráfico aranha dos atributos sensoriais das amostras de néctares de camu-camu em seus diferentes tratamentos ao longo do período de armazenamento, que representa modelo multidimensional das amostras (Figura 2). 


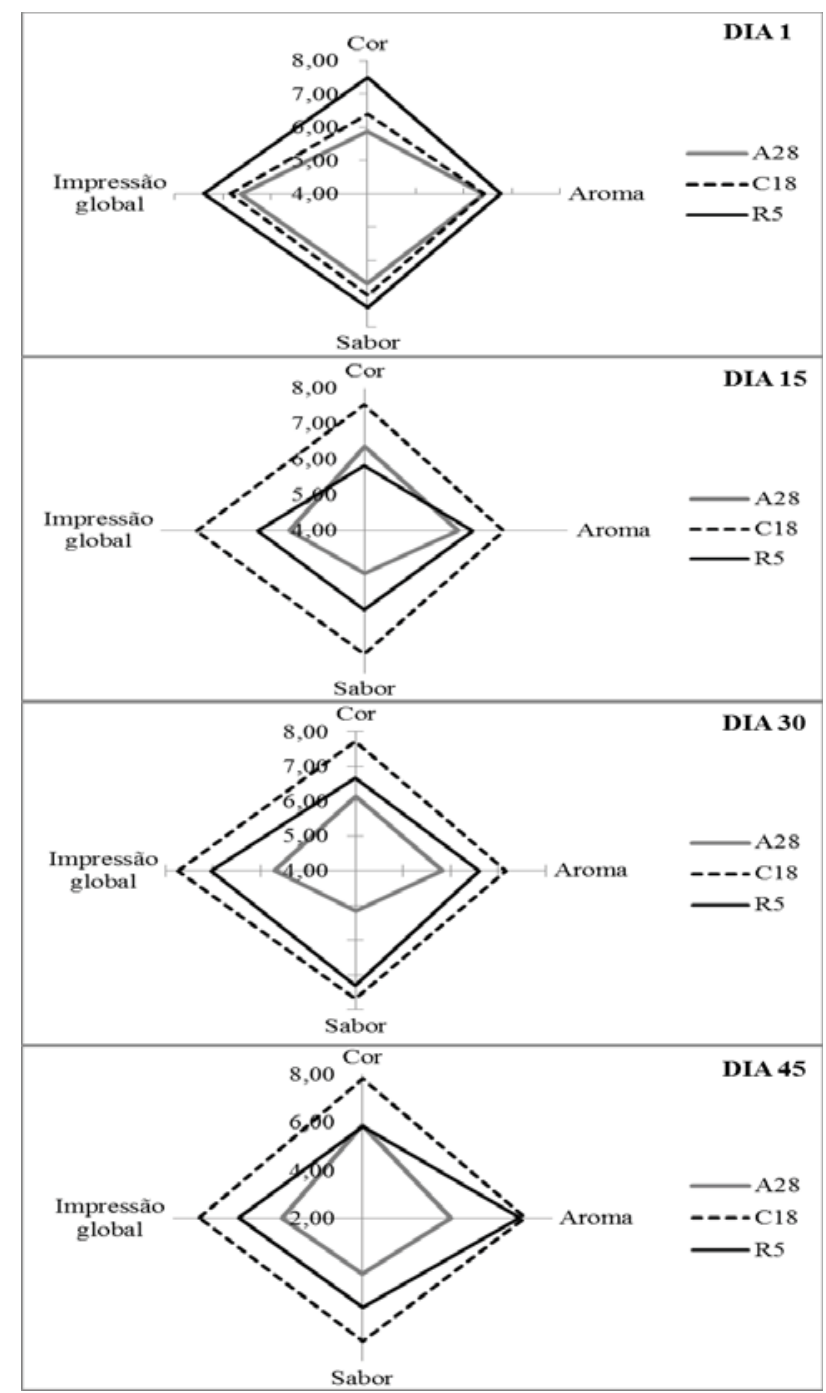

\section{FIGURA 2 - SIMILARIDADES E DIFERENÇAS ENTRE OS NÉCTARES DE CAMU-CAMU ELABORADOS A PARTIR DE POLPA PASTEURIZADA PÓS-EMBALAGEM, ARMAZENADAS SOB DIFERENTES TEMPERATURAS}

A28 = polpa de camu-camu armazenada a $28{ }^{\circ} \mathrm{C} ; \mathrm{C} 18$ = polpa de camu-camu armazenada sob congelamento $\left(-18{ }^{\circ} \mathrm{C}\right)$; $\mathrm{R} 5=$ polpa de camu-camu armazenada sob refrigeração $\left(5^{\circ} \mathrm{C}\right)$.

No primeiro dia de avaliação sensorial dos néctares de polpa de camu-camu, a amostra elaborada com polpa refrigerada (R5) alcançou as maiores notas para os atributos cor, aroma sabor e impressão global. As demais amostras avaliadas (C18 e A28) demonstraram o mesmo comportamento, mas com valores inferiores. Aos 45 dias de armazenamento, o néctar $\mathrm{C} 18$ revelou as melhores características sensoriais para todos os atributos avaliados. A partir do $30^{\circ}$ dia de armazenamento, as polpas R5 e C18 mantiveram suas características sensoriais semelhantes, porém a R5 mostrou valores inferiores para todos os atributos. Apenas no $45^{\circ} \mathrm{dia}$, as duas amostras apresentaram o mesmo comportamento para o atributo aroma.

A partir do período de 15 dias de armazenamento, a amostra A28 recebeu menores notas para sabor, impressão global e aroma, comportamento que se manteve até o final do experimento.

\subsection{AVALIAÇÃO DAS CORRELAÇÕES ENTRE OS ATRIBUTOS SENSORIAIS}

Constatou-se por meio dos coeficientes de correlação, que o atributo sabor do néctar de camu-camu se correlacionou fortemente e de forma positiva $(>0,70)$ com os atributos aroma, cor e impressão global. $O$ atributo impressão global também apresentou correlação forte e positiva com os 
atributos aroma e cor (Tabela 2). Esse resultado indica que quando os atributos sabor ou impressão global recebem notas elevadas dos julgadores, os demais atributos também são bem aceitos.

\section{TABELA 2 - COEFICIENTES DE CORRELAÇÃO PARA OS ATRIBUTOS AVALIADOS NAS AMOSTRAS DE NÉCTAR DE CAMU-CAMU ELABORADO A PARTIR DE POLPA PASTEURIZADA PÓS-EMBALAGEM, ARMAZENADA EM DIFERENTES TEMPERATURAS}

Atributos

Coeficientes de correlação

$\begin{array}{cc}\text { Aroma - Sabor } & 0,84 \\ \text { Aroma - Impressão Global } & 0,87 \\ \text { Cor - Sabor } & 0,72 \\ \text { Cor - Impressão Global } & 0,78 \\ \text { Sabor - Impressão Global } & 0,98\end{array}$

\subsection{ANÁLISE DOS COMPONENTES PRINCIPAIS ENTRE AS AMOSTRAS E OS ATRIBUTOS SENSORIAIS}

Dois componentes principais foram extraídos do conjunto total de dados, explicando 95,26 \% da variância. O primeiro componente principal (PC1) explicou 85,59 \% da variância estatística mediante valores negativos para impressão global, cor, aroma e sabor. O segundo componente principal (PC2) explicou 9,67 \% da variância estatística ao apresentar valores positivos para cor, impressão global e aroma, bem como valores negativos para sabor (Figura 3).

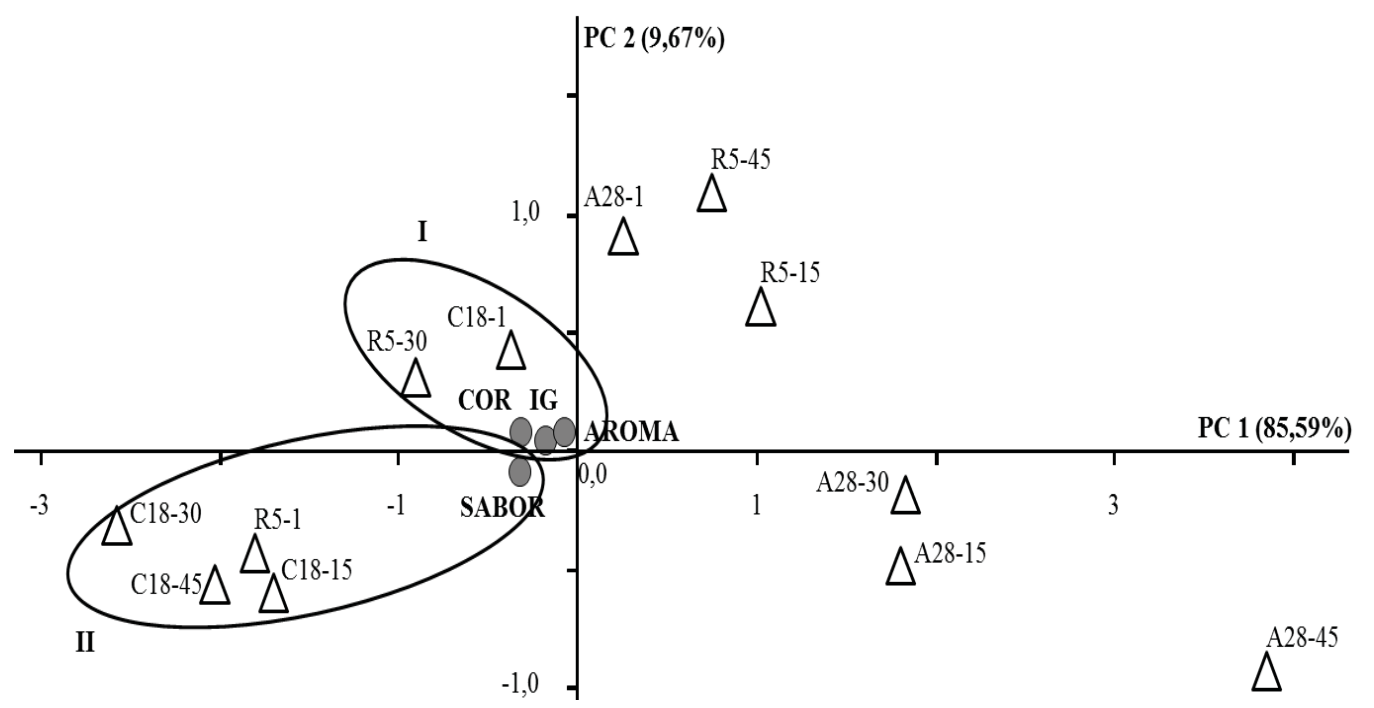

FIGURA 3 - REPRESENTAÇÃO GRÁFICA DOS COMPONENTES PRINCIPAIS REFERENTES AOS ATRIBUTOS SENSORIAIS AVALIADOS NAS AMOSTRAS DE NÉCTAR DE CAMU-CAMU ELABORADO A PARTIR DE POLPA PASTEURIZADA PÓS-EMBALAGEM, ARMAZENADAS EM DIFERENTES TEMPERATURAS

$\mathrm{PC}=$ Componente principal; $\mathrm{x}-\mathrm{y}=$ amostra-período, isto é, amostras: $\mathrm{A} 28$ = polpa de camu-camu armazenada a $28{ }^{\circ} \mathrm{C}$; $\mathrm{C} 18$ = polpa de camu-camu armazenada sob congelamento $\left(-18^{\circ} \mathrm{C}\right) ; \mathrm{R} 5=$ polpa de camu-camu armazenada sob refrigeração $\left(5^{\circ} \mathrm{C}\right)$ e períodos 1-15-30-45 dias de armazenamento.

Os dois componentes principais de todas as amostras foram representados em gráfico, sendo possível verificar a divisão de dois grupos de acordo com o tempo de armazenamento de cada uma (Figura 3). O grupo I, que contém as amostras de néctar de camu-camu elaboradas com 
a polpa refrigerada por 30 dias e a congelada no primeiro dia, foi fortemente caracterizado pelos atributos aroma, impressão global e cor, confirmando os resultados obtidos na análise de diferença de médias e correlação. Ao grupo II pertencem as amostras de néctar de camu-camu produzidas com polpas congeladas nos períodos 15, 30 e 45 dias de armazenamento, bem como a polpa refrigerada no primeiro dia, as quais foram fortemente caracterizadas pelo atributo sabor (Figura 3). Esses dados confirmam os anteriores, justificando a boa aceitação sensorial da polpa R5 no período 1 e o fato da polpa congelada ter alcançado maior aceitação sensorial nos demais períodos avaliados devido ao seu sabor agradável.

\section{CONCLUSÃo}

A polpa refrigerada e a mantida à temperatura ambiente foram bem aceitas, porém é necessário acrescentar corantes e realçadores de sabor, de preferência naturais, à polpa mantida à temperatura ambiente para manter a cor rosa e o sabor característico do fruto.

A polpa mantida à temperatura ambiente pode ser comercializada no mercado com perspectivas de sucesso devido à boa aceitação pelos consumidores, o que é útil para a indústria por ser uma tecnologia mais barata, a qual não utiliza a cadeia do frio.

A temperatura ambiente utilizada para o armazenamento da polpa foi abusiva $\left(28^{\circ} \mathrm{C}\right)$, o que pode ter propiciado degradação mais rápida dos compostos. Há a alternativa de conservação dessa polpa em temperaturas menores, ainda consideradas como temperatura ambiente, não sendo necessária a refrigeração.

O néctar de camu-camu produzido com a polpa pasteurizada pós-embalagem e mantida sob congelamento apresentou as melhores características sensoriais. Tal produto, além de bem aceito pelos julgadores foi fortemente caracterizado pelo sabor.

\section{ABSTRACT \\ SENSORY QUALITY OF CAMU-CAMU NECTAR PRODUCED WITH PASTEURIZED PULP POST-PACKAGE STORED AT DIFFERENT TEMPERATURES}

The objective of this study was to evaluate the sensory quality of pasteurized camu-camu pulp after packaging using high-barrier plastic bags in cold storage $\left(5^{\circ} \mathrm{C}\right)$, frozen $\left(-18{ }^{\circ} \mathrm{C}\right)$ and room temperature $\left(28^{\circ} \mathrm{C}\right)$ for 45 days. The samples were pasteurized in a tank heated to $75^{\circ} \mathrm{C}$ for $1 \mathrm{~min}$ and cooled immediately. The overall acceptance of the nectars were very good, and they received evaluations of "really liked" for the frozen pulp and "liked moderately" for the pulps that were refrigerated and kept at room temperature. The commercialization of camu-camu pulp offers potential but the fruit contains a high level of ascorbic acid and its consumption in fresh form is limited due to this acidity. Camu-camu pulp can be used in the preparation of food drinks, ice cream, jelly, candy and liqueurs.

KEY-WORDS: Myrciaria dubia; NECTARS; THERMAL PROCESSING; POLYPROPYLENE DIFFERENTIATED.

\section{REFERÊNCIAS}

1 ARÉVALO, R.P.; KIECKBUSCH, T.G; QUAST, L.B. Viscosidad aparente de la pulpa de camu-camu (Myrciaria dubia) in natura y con pré-tratamiento térmico. In: CONGRESO INTERAMERICANO DE INGENIERÍA QUÍMICA, 22., Buenos Aires, 2006. Anais... Buenos Aires, Argentina: Asociación Argentina de Ingenieros Químicos, 2006.

2 ARRUDA, A. da S.; LIMA, R.G. de; SILVA, R.M. da; PEIXOTO, N. Desenvolvimento do camu-camu (Myrciaria dubia) em diferentes substratos nas condições de Ipameri-GO. Enciclopédia Biosfera, Goiânia, v.7, n.12, p.1-7, 2011.

3 Association of Official Analytical Chemists (AOAC). Official methods of analysis of AOAC International. $17^{\text {th }}$ ed. Arlington, 2000.

4 BASTOS, C.T.R.M.; LADEIRA, T.M.S.; ROGEZ, H.; PENA, R.S. Estudo da eficiência da pasteurização da polpa de taperebá (Spondias mombin). Alimentos e Nutrição, Araraquara, v.19, n.2, p.123-131, 2008. 
5 BIOCONTROL. 1-2 Test ${ }^{\circledR}$. Disponível em: <http://biocontrolsys.com/products/view/test>. Acesso em: 22/08/2013.

6 BRASIL. Agência Nacional de Vigilância Sanitária. RDC no 12, de 2 de janeiro de 2001. Aprova o regulamento técnico sobre padrões microbiológicos para alimentos. Disponível em: < http://portal.anvisa.gov.br/wps/wcm/connect/ a47bab8047458b909541d53fbc4c6735/RDC_12_2001.pdf?MOD=AJPERES>. Acesso em: 03/07/2014.

7 BRUNINI, M.A.; DURIGAN, J.F.; OLIVEIRA, A.L. Avaliação das alterações em polpa de manga 'Tommy-Atkins' congeladas. Revista Brasileira de Fruticultura, Jaboticabal, v.24, n.3, p.651-653, dez. 2002.

8 COCHRAN, W.G.; COX, G.M. Experimental designs. $2^{\text {nd }}$ ed. London: John Wiley, 1992. 611 p.

9 FRANCO, M.R.B.; SHIBAMOTO, T. Volatile composition of some Brazilian fruits: umbu-caja, camu-camu (Myrciaria dubia), araça-boi (Eugenia stipitata), and cupuaçu (Theobroma grandiflorum). Journal of Agricultural Food Chemistry, v.48, p.1263-1265, mar./2000.

10 GARCIA, E.E.C.; PADULA, M.; SARANTÓPOULOS, C.I.G.L. Embalagens plásticas: propriedades de barreira. Campinas: ITAL, 1989. 44 p.

11 HARPER, A.M.; KOWALSKI, B.R. Individualized differences scaling, the analysis of independent sets of measurements. An application in environmental analytical chemistry. Analytical Letters, New York, v.12, n.7, p.693-712, 1979.

12 Instituto Nacional de Pesquisas da Amazônia (INPA). Cultivo do camu-camu. Disponível em: <http://www.inpa.gov.br/ cpca/areas/camu-camu.html>. Acesso em: 10/08/2011.

13 MAEDA, R.N.; PANTOJA, L.; YUYAMA,L.K.O.; CHAAR, J.M. Determinação da formulação e caracterização do néctar de camu-camu (Myrciaria Dubia Mcvaugh). Ciência e Tecnologia de Alimentos, v.26, n.1, p.70-74, jan./mar. 2006.

14 MAEDA, R.N.; PANTOJA L.; YUYANA, L.K.O.; CHAAR, J.M. Estabilidade de ácido ascórbico e antocianinas em néctar de camu-camu Myrciaria dubia (H. B. K.) Mcvaugh. Ciência e Tecnologia de Alimentos, v.27, n.2, p.313-316, abr./jun. 2007.

15 MATTIETO, R.D.A.; LOPES, A.D.; MENEZES, H.C. de. Estabilidade de néctar misto de cajá e umbu. Ciência e Tecnologia de Alimentos, v.27, n.3, p.456-463, jul./set, 2007.

16 McCUNE, B.; MEFFORD, M.J. PC-ORD: multivariate analysis of ecological data: version 4 for Windows. S.I.: MjM Software Design, 1999.

17 MEILGAARD, M.; CIVILLE, G.V.; CARR, B.T. Sensory evaluation techniques. $3^{\text {rd }}$ ed. (New York): CRC Press LLC, 1999.

18 NEVES, L.C.; BENEDETTE, R.M.; SILVA, V.X.; PRILL, M.A.S.; VIEITES, R.L. Produção de polpas de mangas Tommy Atkins, na Amazônia setentrional, através da aplicação de preservativos e da pasteurização. Revista Brasileira de Fruticultura, Cruz das Almas, v.29, n.3, p.576-582, 2007.

19 OLIVEIRA, E.A.; BORGES, S.V.; FURTADO, A.A.L.; MODESTA, R.C.D.; GODOY, R.O. Heat processing (HTST) of umbu (Spondias tuberosa Arruda Câmara) pulp. Ciência e Tecnologia de Alimentos, São Paulo, v.31, n.4, p.923-928, 2011.

20 SILVA, F.M.; SILVA, C.L.M. Quality evaluation of cupuaçu (Theobroma grandiflorum) purée after pasteurization and during storage. Food Science and Technology International, London, v.6, n.1, p.53-58, 2000.

21 SILVA, R.A. et al. Néctar de caju adoçado com mel de abelha: desenvolvimento e estabilidade. Ciência e Tecnologia de Alimentos, v. 28, n.2, p.348-354, abr./jun. 2008.

22 SILVA, G.M. Degradação da antocianina e qualidade sensorial de polpa de juçara (Euterpe edulis) embalada e submetida à pasteurização. 2012. 98 f. Dissertação (Mestrado em Ciência e Tecnologia de Alimentos), Escola Superior de Agricultura "Luiz de Queiroz", Piracicaba, 2012

23 SCARMINIO, I.S. Desenvolvimento de um sistema quimiométrico para microcomputadores e algumas aplicações. 1989. 124 f. Tese (Doutorado em Química) - Instituto de Química, Universidade Estadual de Campinas, Campinas, 1989.

24 SOUNIS, E. Bioestatística: princípios fundamentais, metodologia estatística, aplicação às ciências biológicas. 2. ed. São Paulo: McGraw-Hill, 1975. SOUSA, M.A.C.; YUYAMA, L.K.O.; AGUIAR, J.P.L.; PANTOJA, L. Suco de açaí (Euterpe oleracea Mart.): avaliação microbiológica, tratamento térmico e vida-de-prateleira. Acta Amazonica, Manaus, v.36, n.4, p.483-496, 2006. Statistical Analysis System Institute. SAS/QC software: usage and reference (version 9.2). Cary, NC, 2005. 1 CDROM.

\section{AGRADECIMENTOS}

Os autores agradecem à Fundação de Amparo à Pesquisa do Estado de São Paulo - FAPESP (processo $n^{\circ}$ 2010/15372-2) pelo apoio na realização do experimento 